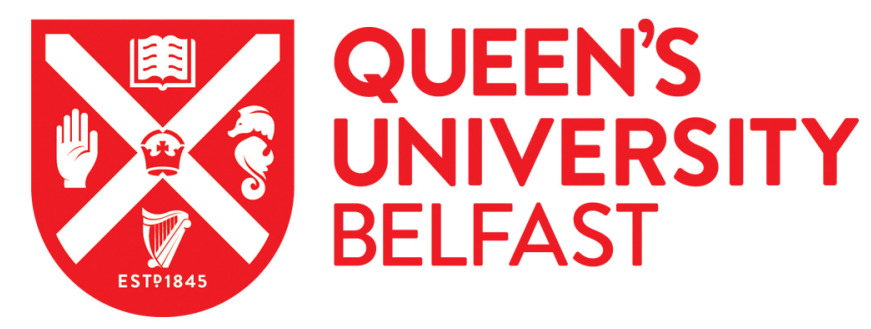

\title{
Analysis of KRAS, NRAS, BRAF, PIK3CA and TP53 mutations in a large prospective series of locally advanced rectal cancer patients
}

\author{
Sclafani, F., Hulkki Wilson, S., Cunningham, D., Gonzalez De Castro, D., Kalaitzaki, E., Begum, R., \\ Wotherspoon, A., Capdevila, J., Glimelius, B., Roselló, S., Thomas, J., Tait, D., Brown, G., Oates, J., \& Chau, I. \\ (2019). Analysis of KRAS, NRAS, BRAF, PIK3CA and TP53 mutations in a large prospective series of locally
} advanced rectal cancer patients. International Journal of Cancer. https://doi.org/10.1002/ijc.32507

Published in:

International Journal of Cancer

\section{Document Version:}

Peer reviewed version

Queen's University Belfast - Research Portal:

Link to publication record in Queen's University Belfast Research Portal

Publisher rights

( 2019 UICC. This work is made available online in accordance with the publisher's policies. Please refer to any applicable terms of use of the publisher.

\section{General rights}

Copyright for the publications made accessible via the Queen's University Belfast Research Portal is retained by the author(s) and / or other copyright owners and it is a condition of accessing these publications that users recognise and abide by the legal requirements associated with these rights.

Take down policy

The Research Portal is Queen's institutional repository that provides access to Queen's research output. Every effort has been made to ensure that content in the Research Portal does not infringe any person's rights, or applicable UK laws. If you discover content in the Research Portal that you believe breaches copyright or violates any law, please contact openaccess@qub.ac.uk. 


\section{Analysis of $K R A S, N R A S, B R A F, P I K 3 C A$ and $T P 53$ mutations in a large prospective series of locally advanced rectal cancer patients}

Francesco Sclafani ${ }^{1}$, Sanna Hulkki Wilson ${ }^{2}$, David Cunningham ${ }^{1}$, David Gonzalez De Castro $\mathrm{D}^{2}$, Eleftheria Kalaitzaki ${ }^{3}$, Ruwaida Begum ${ }^{1}$, Andrew Wotherspoon ${ }^{4}$, Jaume Capdevila ${ }^{5}$, Bengt Glimelius ${ }^{6}$, Susana Roselló ${ }^{7}$, Janet Thomas ${ }^{1}$, Daina Tait ${ }^{8}$, Gina Brown ${ }^{9}$, Jacqui Oates ${ }^{1}$, Ian $\mathrm{Chau}^{1}$

\section{Authors' affiliations:}

${ }^{1}$ Department of Medicine, The Royal Marsden NHS Foundation Trust, London and Surrey, United Kingdom

${ }^{2}$ Department of Molecular Diagnostics, Centre for Molecular Pathology, The Royal Marsden NHS Foundation Trust, London and Surrey, United Kingdom

${ }^{3}$ Department of Clinical Research \& Development, The Royal Marsden NHS Foundation Trust, London and Surrey, United Kingdom

${ }^{4}$ Department of Histopathology, The Royal Marsden NHS Foundation Trust, London and Surrey, United Kingdom

${ }^{5}$ Department of Medical Oncology, Vall d'Hebron Institute of Oncology (VHIO), Barcelona, Spain

${ }^{6}$ Department of Immunology, Genetics and Pathology, Section of Experimental and Clinical Oncology, University of Uppsala, Uppsala, Sweden

7 Department of Haematology and Medical Oncology, Biomedical Research Institute INCLIVA, University of Valencia, Spain

${ }^{8}$ Department of Radiotherapy, The Royal Marsden NHS Foundation Trust, London and Surrey, United Kingdom

This article has been accepted for publication and undergone full peer review but has not been through the copyediting, typesetting, pagination and proofreading process which may lead to differences between this version and the Version of Record. Please cite this article as doi: $10.1002 / \mathrm{ijc} .32507$ 
${ }^{9}$ Department of Radiology, The Royal Marsden NHS Foundation Trust, London and Surrey, United Kingdom

\section{Corresponding author:}

Dr Francesco Sclafani

Department of Medicine

The Royal Marsden NHS Foundation Trust Hospital

Sutton, Surrey

SM2 5PT

United Kingdom

dr.sclafani@gmail.com

Tel: +44-(0)2086613156

Fax: +44-(0)208 6439414

\section{Disclosure}

David Cunningham: research funding from Amgen, AstraZeneca, Bayer, Celgene, Clovis, Eli-Lilly, Janssen, Medimmune, Merck, Merrimack, Sanofi, 4SC. Jaume Capdevila: advisory board and speaker roles for Amgen, Merck, Roche, Bayer, Eisai, Sanofi, Exelixis, Adacap, Novartis, Pfizer and Ipsen. Ian Chau: advisory board for Eli-Lilly, Bristol Meyers Squibb, MSD, Bayer, Roche, Merck-Serono, Five Prime Therapeutics, AstraZeneca, Oncologie International, Pierre Fabre; research funding from Eli-Lilly, Janssen-Cilag, Sanofi Oncology, Merck-Serono; honorarium from Eli-Lilly. All other authors have declared no conflicts of interest. 
Short title: KRAS, NRAS, BRAF, PIK3CA and TP53 in rectal cancer

Keywords: KRAS, NRAS, BRAF, PIK3CA, TP53, rectal cancer

\section{Abbreviations:}

CE-SSCA: capillary electrophoresis-single strand conformational analysis. CI: confidence intervals. EGFR: epidermal growth factor receptor. EMVI: extramural venous invasion. HR: hazard ratio. LARC: locally advanced rectal cancer. MMR: mismatch repair. MMS: microsatellite stable. MRI: magnetic resonance imaging. MSI: microsatellite instability. NGS: next-generation sequencing. PCR: polymerase chain reaction. pCR: pathological complete response. PFS: progression-free survival. pTRG: pathological tumour regression grade. OS: overall survival.

\section{Novelty and Impact}

This article reports the incidence, heterogeneity and clinical significance of $K R A S, N R A S$, $B R A F, P I K 3 C A$ and TP53 mutations in a prospective series of 210 non-metastatic rectal cancer patients. Main findings include an association between TP53 mutations and poor pathological regression grade after neoadjuvant treatment, and worse survival outcome among patients with tumours harbouring concomitant TP53 and RAS mutations. Upon confirmation, these results may be used for patient stratification in future clinical trials. 


\begin{abstract}
Little information is available on the clinical significance of cancer-related genes such as KRAS, NRAS, BRAF, PIK3CA and TP53 in non-metastatic rectal cancer. We investigated mutations of these genes in a large prospective series of locally advanced rectal cancer (LARC) patients who were recruited into two phase II trials. Mutational analyses were performed with diagnostically validated methods including polymerase chain reaction, capillary electrophoresis-single strand conformational analysis, Sanger sequencing and nextgeneration sequencing. Associations between single or multiple gene mutations and clinicopathological characteristics and treatment outcomes were explored. 210/269 (78\%) patients were assessable. Mutations of KRAS, NRAS, BRAF, PIK3CA and TP53 occurred in 43\%, 9\%, $4 \%, 9 \%$ and $60 \%$ of patients, respectively. Concordance between paired biopsy and resection specimens was $82 \%$ for $K R A S, 95 \%$ for $N R A S, 99 \%$ for $B R A F, 96 \%$ for $P I K 3 C A$ and $63 \%$ for TP53. TP53 mutations were associated with extramural venous invasion on baseline MRI (78\% vs $65 \%, \mathrm{p}=0.04)$, good pathological tumour regression $(36 \%$ vs $23 \%, \mathrm{p}=0.05)$ and a trend towards a better 5-year progression-free survival ( $74 \%$ vs 60\%, HR 1.59, p=0.06). Patients with tumours harbouring mutation of TP53 and either KRAS or NRAS (32\%) had a worse 5-year progression-free survival than those with TP53/KRAS/NRAS wild-type tumours (54\% vs $72 \%$, HR $1.75, \mathrm{p}=0.02)$. In univariate analysis $B R A F$ mutation predicted poor 5 -year overall survival only among patients treated without cetuximab (20\% vs $73 \%$, HR 3.29, $\mathrm{p}=0.03$ ). This is one of the largest biomarker studies in a prospective, largely homogeneous, LARC population. Our findings are hypothesis-generating and require validation in independent series.
\end{abstract}




\section{Introduction}

In early stage colon cancer and metastatic colorectal cancer molecular tests are routinely performed to capture useful information on tumour biology and guide treatment decisions. Testing for microsatellite instability (MSI)/mismatch repair (MMR) deficiency allows for identification of good prognosis stage II colon cancer patients who do not require adjuvant chemotherapy while mutational analysis of $K R A S, N R A S$ and $B R A F$ provides a tool to predict resistance to anti-EGFR monoclonal antibodies and prognosis in stage IV colorectal tumours.

In contrast, the management of non-metastatic rectal cancer still lacks biomarkers that could refine prognostication and treatment response prediction as currently provided by conventional clinical, pathological and imaging factors (1). While important advances have been made in the definition of risk categories and implementation of risk-stratified treatment approaches (2-9), much still needs to be done to capture the underlying inter-individual tumour heterogeneity and to identify molecular determinants of treatment responsiveness or resistance. As a result, therapeutic algorithms for non-metastatic rectal cancer remain suboptimal and different outcomes are generally seen among patients who share similar clinico-pathological risk features and are elected to receive the same treatment.

Retrospective analyses of clinical trials suggest that KRAS mutation (especially for left-sided and rectal tumours) and $B R A F$ mutation (at least in microsatellite stable [MMS] or MMR proficient tumours) predict poor prognosis of colon cancer patients (10-13) while PIK3CA and TP53 mutation are associated with increased risk of local recurrence and resistance to radiotherapy, respectively $(14,15)$. Nevertheless, data are scant overall and more studies are needed. Furthermore, there is very limited information regarding the prognostic/predictive 
value of these genetic alterations when simultaneously detected in the same tumour. Therefore, we investigated baseline clinical characteristics, treatment outcome and survival of a large prospective series of LARC patients according to the mutational status of five genes including KRAS, NRAS, BRAF, PIK3CA and TP53.

\section{Material and Methods}

\section{Patient population}

PAN-EX was a pooled analysis of two phase II trials sponsored by The Royal Marsden NHS Foundation Trust (16). EXPERT was a single-centre, single-arm, phase II trial (2001-2005) while EXPERT-C was an international, multicentre, randomised phase II trial (2005-2008) $(17,18)$. Main eligibility criteria for both studies included non-metastatic rectal cancer with at least one of the following high-risk features on high-resolution pelvic MRI at baseline: tumour $<1 \mathrm{~mm}$ of the mesorectal fascia, extramural invasion $>5 \mathrm{~mm}(\mathrm{~T} 3 \mathrm{c} / \mathrm{d}), \mathrm{T} 4$ stage, T3 tumour at/below levator muscles. Additional high-risk imaging features for eligibility included N2 stage (EXPERT) and extramural venous invasion (EMVI) (EXPERT-C) $(17,18)$

\section{Treatment}

All patients were treated with 4 cycles of neoadjuvant CAPOX chemotherapy followed by capecitabine-based chemo-radiotherapy (54 Gy in EXPERT and 50.4 Gy in EXPERT-C). Surgery was performed according to the principles of total mesorectal excision 4-6 weeks after completion of chemo-radiotherapy. Four cycles of adjuvant chemotherapy (capecitabine in EXPERT and CAPOX in EXPERT-C) were also administered. Patients in the EXPERT-C study were randomised in a 1:1 ratio to receive cetuximab in combination with neoadjuvant chemotherapy, chemoradiotherapy and adjuvant chemotherapy. Follow-up was carried out for 5 years after surgery $(17,18)$. 


\section{Mutation analyses}

All mutation analyses were performed in a central laboratory (Centre for Molecular Pathology, The Royal Marsden Hospital NHS Foundation Trust) on DNA extracted from formalin-fixed, paraffin-embedded tissue sections from diagnostic biopsy and/or posttreatment primary resection samples. Given that the PAN-EX study analysed all mutational data that were obtained over time from samples of patients included in the EXPERT and EXPERT-C trials, different analytic techniques were used. In the EXPERT-C study, analysis of $K R A S$ (exon 2 and 3) and BRAF (exon 15) was performed prospectively using the INFINITI platform (AutoGenomics, Vista, CA), as per the manufacturer's instructions. Mutations in PIK3CA exon 9 and 20 and NRAS exon 3 were screened for by capillary electrophoresis-single strand conformational analysis (CE-SSCA) followed by bi-directional Sanger sequencing. Mutations in exon 4 of KRAS and exons 2 and 4 of NRAS were screened for by bi-directional Sanger sequencing. All mutations detected were confirmed on an independent PCR and sequencing analysis (17-19). TP53 mutational analysis (exon 4-9) was performed by CE-SSCA and bi-directional Sanger sequencing analysis performed on an independent PCR (20). In the EXPERT study, mutations in KRAS (exon 2-4), NRAS (exon 24), BRAF (exon 15), PIK3CA (exon 9 and 20) and TP53 (exon 4-11) were retrospectively screened for using a next-generation sequencing (NGS) panel which was developed in house (Centre for Molecular Pathology, The Royal Marsden Hospital NHS Foundation Trust) and subsequently validated for routine clinical application. All the analyses were performed by investigators who were blinded to the clinical data.

\section{Comparison of NGS against other sequencing techniques}


Given the use of different sequencing techniques between EXPERT and EXPERT-C, 45 samples from 37 EXPERT-C patients with available tumour tissue were also analysed with the same NGS panel which was used for EXPERT patients. Concordance rates were as follows: $89 \%$ (40/45) for KRAS (4 new mutations detected while 1 mutation missed), $98 \%$ (44/45) for NRAS (1 mutation missed), 100\% (45/45) for BRAF, 98\% (44/45) for PIK3CA (1 new mutation detected) and 91\% (41/45) for TP53 (3 new mutations detected while 1 mutation missed; a second mutation was missed in 2 mutant samples, one each by CE-SSCA and NGS).

\section{Outcome measures and statistical analysis}

Only patients who had tumour samples assessable for genetic analyses were included in this study. Pathological tumour regression grade (pTRG) was assessed (prospectively in EXPERT-C and retrospectively in EXPERT) by local independent pathologists according to Dworak et al (21). For the purpose of this analysis, good tumour regression corresponded to pTRG 3 or 4 while pTRG $0-2$ indicated poor tumour regression. Pathological complete response (pCR) was defined as the absence of viable tumour cells in the tumour bed and resected lymph nodes. Progression-free survival (PFS) and overall survival (OS) were calculated from trial start date to date of recurrence and death, respectively. Patients alive and without evidence of tumour progression were censored at last follow-up. Patients who died without tumour progression were censored at the time of death.

All pooled analyses were stratified by treatment arm and trial. For the PFS and OS endpoints the Kaplan-Meier method was used and median survival along with 95\% confidence intervals (CI) were estimated according to mutational status. Cox proportional hazards regression models were fitted to produce the estimated hazard ratios (HR) and survival probabilities. 
Univariate analyses were performed to examine the crude relationship between marker and PFS/OS. In view of the exploratory nature of the study, a multivariable model was fitted initially with standard clinico-pathological variables while $K R A S, N R A S, B R A F, P I K 3 C A$, and TP53 were added in a forward selection procedure. Prognostic variables were retained in the model if they demonstrated significance at the $\leq 10 \%$ level (i.e., $p \leq 0.10$ ). Logistic regression was used for the dichotomised endpoints (i.e., pTRG 3-4 vs pTRG 0-2 and pCR vs non-pCR).

Data are available upon request.

\section{Regulatory approval}

EXPERT and EXPERT-C were approved by the relevant National Regulatory Agencies and Research Ethics Committee. All patients provided written informed consent. The PAN-EX study was approved by the Committee for Clinical Research at The Royal Marsden NHS Foundation Trust.

\section{Results}

Two-hundred and sixty-nine patients were included in the PAN-EX study (105 from EXPERT and 164 from EXPERT-C) (Supplementary Table 1). Of these, 210 (78\% [58\% and 91\% of the EXPERT and EXPERT-C patient population, respectively]) were assessable for $\geq 1$ biomarker while $59(22 \%)$ were not assessable due to lack of tumour tissue (i.e., archived material not retrievable and/or pCR on resection specimen) or poor quality of the available samples (i.e., low DNA concentration). The majority of assessable patients had only one sample available for analysis (either baseline biopsy or resection sample) while a variable number (ranging from 57 to 71 depending on the biomarker) could be successfully analysed in paired (baseline biopsy and resection) samples (Figure 1). 


\section{Analysis of single mutations}

Mutation rates in biopsy and resection samples are shown in Table 1 (full list of mutations including type and frequency available in Supplementary Table 2). Overall 43\%, 9\%, 4\%, $9 \%$ and $60 \%$ of patients had KRAS, NRAS, BRAF, PIK3CA and TP53 mutant tumours, respectively. Two TP53 mutations co-occurred in 7 cases. The concordance rates between paired specimens was $82 \%$ for $K R A S, 95 \%$ for $N R A S, 99 \%$ for $B R A F, 96 \%$ for $P I K 3 C A$ and $63 \%$ for TP53 (Table 2). No statistically significant associations were observed between mutations and baseline prognostic factors with the only exception of TP53 mutations which were associated with MRI-detected EMVI (78\% in TP53 mutant tumours vs $65 \%$ in TP53 wild-type tumours, $\mathrm{p}=0.04$ ) (Supplementary Tables 3-7).

In the entire study population, patient outcomes did not differ according to the mutational status of KRAS, NRAS, BRAF, or PIK3CA. Numeric differences were found between TP53 wild-type and TP53 mutant patients in terms of pCR (17\% vs 9\%, p=0.08), good tumour regression $(36 \%$ vs $23 \%, \mathrm{p}=0.05)$ and 5-year PFS (74\% vs 60\%, HR 1.59 [95\% CI: 0.98 2.58], $\mathrm{p}=0.06)$ but not for 5-year OS (77\% vs 72\%, HR 1.20 [95\% CI: 0.72-2.00], p=0.48).

(Table 3). The association between TP53 mutation and PFS remained unaltered (HR 1.65 [95\% CI: 0.99-2.75], $\mathrm{p}=0.06)$ after multivariate analysis.

When the analyses were restricted to the group of patients who did not receive cetuximab no statistically significant associations were detected between single gene mutations and patient outcome with the only exception of BRAF and TP53 mutations. The former was associated with a worse 5-year OS (20\% vs 73\%, HR 3.29 [95\% CI: $1.16-9.28], \mathrm{p}=0.03)$ and a trend towards a worse 5-year PFS (20\% vs 66\%, HR 2.54 [95\% CI: 0.91-7.10], p=0.08). The latter 
predicted poor tumour regression $(84 \%$ vs $61 \%, \mathrm{p}=0.01)$. After multivariate analysis, the association between $B R A F$ mutation and OS did not reach statistical significance (HR 2.54 [95\% CI: 0.91-7.13], $\mathrm{p}=0.08)$.

\section{Analysis of mutation combinations}

KRAS or NRAS mutations were detected in $105 / 203$ patients (52\%), KRAS, NRAS or BRAF mutations in 111/202 (55\%), KRAS, NRAS, BRAF or PIK3CA mutations in 114/202 (56\%), KRAS, NRAS, BRAF, PIK3CA or TP53 mutations in 170/205 (83\%). No associations between any of these mutation combinations and either prognostic factors or treatment outcomes were found to be significant at 5\% level association. There were 63 out of 199 patients (32\%) with tumours harbouring mutations of TP53 and either KRAS or NRAS. These had an older age (median age 64.2 vs 60.4 years, $\mathrm{p}=0.02$ ) and different stage distribution at diagnosis (i.e., stage II/III tumours $30 \%$ vs $70 \%, \mathrm{p}=0.01$ ) than patients with TP53/KRAS/NRAS wild-type tumours. While no association was observed between TP53 and KRAS/NRAS mutations and early outcome efficacy measures (i.e., pCR and tumour regression), patients with TP53 and KRAS/NRAS mutant tumours had a worse 5-year PFS than those with TP53/KRAS/NRAS wild-type tumours (54\% vs 72\%, HR 1.75 [95\% CI: 1.10-2.78], p=0.02) (Figure 2). This association remained significant after adjusting for prognostic factors in multivariate analyses (HR 1.74 [1.07-2.85], $\mathrm{p}=0.03$ ). In the group of patients who did not receive cetuximab, none of the mutation combinations was statistically significantly associated with either short- or long-term outcome measures.

\section{Discussion}

In this study we analysed the clinical significance of mutations of five genes, including $K R A S, N R A S, B R A F, P I K 3 C A$ and TP53, in a large prospective series of LARC patients. The 
results of our analysis suggest an association between TP53 mutations and MRI-detected EMVI at baseline and poor tumour regression after neoadjuvant treatment. Furthermore, we found that patients with tumours harbouring concomitant TP53 and RAS mutations had a worse PFS than those with wild-type tumours for either of these genes. Finally, in the group of patients who were treated with chemotherapy and chemoradiotherapy but without cetuximab, BRAF mutations were associated with a worse OS in univariate analysis.

The genes which were selected for this analysis are all known to be biologically and clinically relevant as they play a key role in the processes of colorectal cancer carcinogenesis and tumour progression and in the mechanisms of treatment resistance $(22,23)$. Nevertheless, the bulk of evidence underlying this information consists of studies that were conducted in metastatic colorectal cancer patients while limited data exist for rectal cancer especially in the non-metastatic setting. Therefore, investigation in a largely homogeneous, prospective cohort of LARC patients is warranted. In this regard, PAN-EX (a pooled analysis of two academic phase II rectal cancer trials of similar design, EXPERT and EXPERT-C) provides a unique, valuable platform for exploratory biomarker analyses (16).

TP53 mutations occur in the late phase of the step-wise, colorectal carcinogenesis process and are particularly common among non-hypermutated tumours $(22,24)$. In line with the role of the TP53 pathway in the mechanisms of DNA repair following genotoxic stress, studies have demonstrated an association between TP53 mutations and resistance to radiotherapy (15). This association has been confirmed by the results of our study which are also in line with previous data showing a higher incidence of vascular invasion in TP53 mutant colorectal cancers (25). Interestingly, the improved pathological regression observed in our study among patients with TP53 wild-type tumours translated into numerically higher but not 
statistically significant survival outcomes. While this inconsistency may be secondary to the limited sample size and the relatively low number of survival events, it is also possible that the long-term prognostic effect of TP53 could have been diluted by the inclusion of patients who were treated without cetuximab and in fact accounted for the majority of the study population. Indeed, in a previous exploratory biomarker analysis of the EXPERT-C trial, we showed that TP53 was an independent predictive factor for PFS and OS only in the group of cetuximab-treated patients, possibly due to a selective therapeutic effect of EGFR inhibition on micrometastatic foci of TP53 wild-type tumours $(20,26)$. Therefore, beyond the confirmation of reduced pathological regression of TP53 mutant tumours after neoadjuvant therapy, the findings of our analysis appear to provide further indirect support to the design of prospective trials investigating TP53 as predictive biomarker for cetuximab in LARC.

Mutations of the KRAS gene occur before TP53 mutations in the early stages of the adenomacarcinoma sequence (27). While testing for these genetic aberrations (alongside NRAS mutations) is a routine procedure to select metastatic colorectal cancer patients for treatment with anti-EGFR monoclonal antibodies, the significance of KRAS/NRAS mutations in the setting of non-metastatic rectal cancer is unknown. Data from previous studies are inconsistent. KRAS mutations were associated with a reduced rate of $\mathrm{pCR}$ or worse long-term outcome in some series (28-32) but not in others (33-38). Of note, in a study of 229 rectal cancer patients who had received neoadjuvant chemotherapy either before or after chemoradiotherapy, Chow et al showed that patients who had KRAS/TP53 double mutant tumours achieved a lower rate of pCR compared to patients with non-double mutant tumours $(10 \%$ vs $31 \%, p=0.001)$, this association being likely driven by the negative prognostic effect of KRAS mutations in the same series (29). In our study, we did not find any difference in outcome between patients with KRAS (or KRAS/NRAS) wild-type and KRAS (or KRAS/NRAS) 
mutant tumours. On the other hand, in line with the study by Chow et al, we observed a poorer PFS among patients with tumours harbouring concomitant $R A S$ and TP53 mutations. While, this finding may actually be biased by the reduced response to treatment and poor outcome of TP53 mutant tumours in our series, further investigation of the prognostic role of concurrent RAS and TP53 mutations in future studies may be warranted. In contrast, none of the other mutation combinations which were tested in our study appeared to have any impact on treatment outcome or patient prognosis.

As expected for rectal cancers, mutations of the $B R A F$ gene were detected in a small proportion of our patients (4\%). Bearing in mind that the rarity of this alteration precludes any meaningful analysis, we found an association between $B R A F$ mutations and poor OS which was significant in univariate analysis only and limited to the group of patients treated without cetuximab. The absence of differences between patients with BRAF mutant and $B R A F$ wild-type tumours in terms of pathological tumour regression or PFS suggests that this association may be secondary to the poor prognosis conferred by BRAF mutation after tumour recurrence as previously reported (39). Larger series are certainly needed to clarify the prognostic and predictive value of $B R A F$ mutation in this disease setting. Of note, recent studies have shown that non-V600 BRAF mutations account for $22 \%$ of all BRAF mutations. These occur more frequently in rectal cancers and are associated with more favourable clinico-pathological features and better outcomes than canonical V600 BRAF mutations (40, 41). In our series, approximately one third of $B R A F$ mutations were non-V600 but the small numbers did not allow us to explore any association with clinical data.

Spatial and temporal intra-tumour molecular heterogeneity is a landmark of many malignancies including colorectal cancer $(42,43)$. Studies addressing this phenomenon in 
non-metastatic rectal cancer are limited and results not always consistent (44-48). By analysing a relatively large number of paired tumour tissues from pre-treatment biopsies and post-treatment resection samples we found a high concordance ( $\geq 95 \%)$ for $N R A S, B R A F$ and PIK3CA while the concordance for $K R A S$ and TP53 was lower at $82 \%$ and $63 \%$, respectively. Notably, the vast majority of discordant cases in our series were due to the detection at baseline of mutant clones which were not subsequently detectable on the resection specimens, this likely reflecting an artefact secondary to the reduced tumour cellularity after neoadjuvant treatment. As previously shown, the rate of discordance can be significantly reduced by analysing post-treatment resection samples with more sensitive detection techniques than those used for diagnostic purposes on the pre-treatment biopsy (47). On the other hand, sampling errors may account for the few remaining "false negative" (i.e., wildtype) biopsy cases and highlight the potential value of multiple sampling at baseline as well as further investigation and validation of circulating tumour DNA mutational analyses (49).

The results of our analysis should be interpreted with extreme caution due to a number of limitations. The PAN-EX study was meant to analyse all mutational data that were obtained over time from samples of patients included in two prospective trials, this inevitably resulting in the use of several analytic platforms. Nevertheless, in view of the high concordance rates between NGS and other sequencing techniques (ranging from $89 \%$ for $K R A S$ to $100 \%$ for $B R A F)$ as observed in a small sample of the study population, it is unlikely that this heterogeneity could have significantly affected the final results. While all "false negative" cases by NGS were secondary to the poor quality of the re-tested samples, the "false negative cases" by other sequencing techniques were due to either technical issues or mutations which were below the detection level. Other study limitations include the retrospective analysis, the relatively high proportion of non-assessable patients (especially for the EXPERT study), the 
small number of genes analysed, the limited number of exons tested for each gene, and some treatment heterogeneity between the two study populations. Furthermore, in light of the multiple testing some of the statistically significant associations between mutated genes and clinico-pathological characteristics or treatment outcomes could be random effects. It should be noted that the PAN-EX study was originally designed as an exploratory analysis and no formal a-priori sample size calculation was made. Despite the relatively large population, this study does not have sufficient power to detect meaningful effects and the lack of sufficient events/observations is confirmed by the very wide confidence intervals even in the presence of results which ultimately met the criteria for statistical significance. Larger studies of independent series are needed to support our findings which remain hypothesis-generating. Nevertheless, useful insights can be obtained from this analysis, which is one of the largest of its kind, including a better understanding of the potential clinical utility of testing LARC patients for genetic variants which are commonly evaluated in routine oncology practice.

There is no doubt that refinement of currently adopted risk-stratified treatment strategy for non-metastatic rectal cancer is needed and unlikely to happen without the identification and validation of clinically actionable molecular alterations. Studies providing a comprehensive and integrated molecular characterisation of rectal tumours and exploring clinical correlations in relation to prognosis and response to treatment are highly desirable and likely to shape the future treatment landscape of this disease. 


\section{References}

1. Glynne-Jones R, Wyrwicz L, Tiret E, et al. Rectal cancer: ESMO clinical practice guidelines for diagnosis, treatment and follow-up. Ann Oncol 2018; 29(Suppl 4):iv263.

2. Brown G, Radcliffe AG, Newcombe RG, et al. Preoperative assessment of prognostic factors in rectal cancer using high-resolution magnetic resonance imaging. $\mathrm{Br} J$ Surg 2003;90:355-64.

3. Blomqvist L, Glimelius B. The "good", the "bad", and the "ugly" rectal cancers. Acta Oncol 2008;47:5-8.

4. Engelen SM, Maas M, Lahaye MJ, et al. Modern multidisciplinary treatment of rectal cancer based on staging with magnetic resonance imaging leads to excellent local control, but distant control remains a challenge. Eur J Cancer 2013;49:2311-20.

5. Taylor FG, Quirke P, Heald RJ, et al. Preoperative high-resolution magnetic resonance imaging can identify good prognosis stage I, II, and III rectal cancer best managed by surgery alone: a prospective, multicenter, European study. Ann Surg 2011;253:711-9.

6. Patel UB, Taylor F, Blomqvist L, et al. Magnetic resonance imaging-detected tumor response for locally advanced rectal cancer predicts survival outcomes: MERCURY experience. J Clin Oncol 2011;29:3753-60. 
7. Schrag D, Weiser MR, Goodman KA, et al. Neoadjuvant chemotherapy without routine use of radiation therapy for patients with locally advanced rectal cancer: a pilot trial. $J$ Clin Oncol 2014;32:513-8.

8. van der Valk MJM, Hilling DE, Bastiaannet E, et al. Long-term outcomes of clinical complete responders after neoadjuvant treatment for rectal cancer in the International Watch \& Wait Database (IWWD): an international multicentre registry study. Lancet 2018;391:2537-45.

9. Sclafani F, Brown G. Extramural venous invasion (EMVI) and tumour regression grading (TRG) as potential prognostic factors for risk stratification and treatment decision in rectal cancer. Curr Colorectal Cancer Rep 2016; doi 10.1007/s11888-016-0319-4.

10. Hutchins G, Southward K, Handley K, et al. Value of mismatch repair, KRAS, and BRAF mutations in predicting recurrence and benefits from chemotherapy in colorectal cancer. $J$ Clin Oncol 2011;29:1261-70.

11. Sinicrope FA, Mahoney MR, Yoon HH, et al. Analysis of molecular markers by anatomic tumor site in stage III colon carcinomas from adjuvant chemotherapy trial NCCTG N0147 (Alliance). Clin Cancer Res 2015;21:5294-304.

12. Sinicrope FA, Mahoney MR, Smyrk TC, et al. Prognostic impact of deficient DNA mismatch repair in patients with stage III colon cancer from a randomized trial of FOLFOXbased adjuvant chemotherapy. J Clin Oncol 2013;31:3664-72.

13. Taieb J, Le Malicot K, Shi Q, et al. Prognostic value of BRAF and KRAS mutations in MSI and MSS stage III colon cancer. J Natl Cancer Inst 2016;109.

14. He Y, Van't Veer LJ, Mikolajewska-Hanclich I, et al. PIK3CA mutations predict local recurrences in rectal cancer patients. Clin Cancer Res 2009;15:6956-62. 
15. Chen $\mathrm{M}-\mathrm{B}, \mathrm{Wu} \mathrm{X}-\mathrm{Y}, \mathrm{Yu} \mathrm{R}$, et al. P53 status as a predictive biomarker for patients receiving neoadjuvant radiation-based treatment: a meta-analysis in rectal cancer. PLoS One 2012;7:e45388.

16. Sclafani F, Brown G, Cunningham D, et al. PAN-EX: a pooled analysis of two trials of neoadjuvant chemotherapy followed by chemoradiotherapy in MRI-defined, locally advanced rectal cancer. Ann Oncol 2016;27:1557-65.

17. Chua YJ, Barbachano Y, Cunningham D, et al. Neoadjuvant capecitabine and oxaliplatin before chemoradiotherapy and total mesorectal excision in MRI-defined poor-risk rectal cancer: a phase 2 trial. Lancet Oncol 2010;11:241-8.

18. Dewdney A, Cunningham D, Tabernero J, et al. Multicenter randomized phase II clinical trial comparing neoadjuvant oxaliplatin, capecitabine, and preoperative radiotherapy with or without cetuximab followed by total mesorectal excision in patients with high-risk rectal cancer (EXPERT-C). J Clin Oncol 2012;30:1620-7.

19. Sclafani F, Gonzalez D, Cunningham D, et al. RAS mutations and cetuximab in locally advanced rectal cancer: results of the EXPERT-C trial. Eur J Cancer 2014;50:1430-6.

20. Sclafani F, Gonzalez D, Cunningham D, et al. TP53 mutational status and cetuximab benefit in rectal cancer: 5-year results of the EXPERT-C trial. J Natl Cancer Inst 2014;106.

21. Dworak O, Keilholz L, Hoffman A. Pathological features of rectal cancer after preoperative radiochemotherapy. Int J Colorectal Dis 1997;12:19-23.

22. Goyette MC, Cho K, Fasching CL, et al. Progression of colorectal cancer is associated with multiple tumor suppressor gene defects but inhibition of tumorigenicity is accomplished by correction of any single defect via chromosome transfer. Mol Cell Biol 1992;12:1387-95.

23. De Roock W, Claes B, Bernasconi D, et al. Effects of KRAS, BRAF, NRAS, and PIK3CA mutations on the efficacy of cetuximab plus chemotherapy in chemotherapy- 
refractory metastatic colorectal cancer: a retrospective consortium analysis. Lancet Oncol 2010;11:753-62.

24. Cancer Genome Atlas Network. Comprehensive molecular characterization of human colon and rectal cancer. Nature 2012;487:330-7.

25. Russo A, Bazan V, Iacopetta B, et al. The TP53 colorectal cancer international collaborative study on the prognostic and predictive significance of p53 mutation: influence of tumor site, type of mutation, and adjuvant treatment. J Clin Oncol 2005;23:7518-28.

26. Sclafani F, Gonzalez de Castro D, Cunningham D, et al. Fc $\gamma$ RIIa and Fc $\gamma$ RIIIa polymorphisms and cetuximab benefit in the microscopic disease. Clin Cancer Res 2014;20:4511-9.

27. Cho KR, Vogelstein B. Genetic alterations in the adenoma-carcinoma sequence. Cancer 1992;70:1727-31.

28. Garcia-Aguilar J, Chen Z, Smith DD, et al. Identification of a biomarker profile associated with resistance to neoadjuvant chemoradiation therapy in rectal cancer. Ann Surg 2011;254:486-92.

29. Chow OS, Kuk D, Keskin M, et al. KRAS and combined KRAS/TP53 mutations in locally advanced rectal cancer are independently associated with decreased response to neoadjuvant therapy. Ann Surg Oncol 2016;23:2548-55.

30. Duldulao MP, Lee W, Nelson RA, et al. Mutations in specific codons of the KRAS oncogene are associated with variable resistance to neoadjuvant chemoradiation therapy in patients with rectal adenocarcinoma. Ann Surg Oncol 2013;20:2166-71.

31. Gaedcke J, Grade M, Jung K, et al. KRAS and BRAF mutations in patients with rectal cancer treated with preoperative chemoradiotherapy. Radiother Oncol 2010;94:76-81. 
32. Kohonen-Corish MR, Tseung J, Chan C, et al. KRAS mutations and CDKN2A promoter methylation show an interactive adverse effect on survival and predict recurrence of rectal cancer. Int J Cancer 2014;134:2820-8.

33. Bengala C, Bettelli S, Bertolini F, et al. Prognostic role of EGFR gene copy number and KRAS mutation in patients with locally advanced rectal cancer treated with preoperative chemoradiotherapy. Br J Cancer 2010;103:1019-24.

34. Davies JM, Trembath D, Deal AM, et al. Phospho-ERK and AKT status, but not KRAS mutation status, are associated with outcomes in rectal cancer treated with chemoradiotherapy. Radiat Oncol 2011;6:114.

35. Derbel O, Wang Q, Desseigne F, et al. Impact of KRAS, BRAF and PI3KCA mutations in rectal carcinomas treated with neoadjuvant radiochemotherapy and surgery. BMC Cancer 2013;13:200.

36. Luna-Pérez P, Segura J, Alvarado I, et al. Specific c-K-ras gene mutations as a tumorresponse marker in locally advanced rectal cancer treated with preoperative chemoradiotherapy. Ann Surg Oncol 2000;7:727-31.

37. Zauber NP, Marotta SP, Berman E, et al. Molecular genetic changes associated with colorectal carcinogenesis are not prognostic for tumor regression following preoperative chemoradiation of rectal carcinoma. Int J Radiat Oncol Biol Phys 2009;74:472-6.

38. Demes M, Scheil-Bertram S, Bartsch H, et al. Signature of microsatellite instability, KRAS and BRAF gene mutations in German patients with locally advanced rectal adenocarcinoma before and after neoadjuvant 5-FU radiochemotherapy. J Gastrointest Oncol 2013;4:182-92.

39. Roth AD, Tejpar S, Delorenzi M, et al. Prognostic role of KRAS and BRAF in stage II and III resected colon cancer: results of the translational study on the PETACC-3, EORTC 40993, SAKK 60-00 trial. J Clin Oncol 2010;28:466-74. 
40. Cremolini C, Di Bartolomeo M, Amatu A, et al. BRAF codons 594 and 596 mutations identify a new molecular subtype of metastatic colorectal cancer at favorable prognosis. Ann Oncol 2015;26:2092-7.

41. Jones JC, Renfro LA, Al-Shamsi HO, et al. Non-V600 BRAF mutations define a clinically distinct molecular subtype of metastatic colorectal cancer. J Clin Oncol 2017;35:2624-30.

42. Uchi R, Takahashi Y, Niida A, et al. Integrated multiregional analysis proposing a new model of colorectal cancer evolution. PLoS Genet 2016;12:e1005778.

43. Sottoriva A, Kang H, Ma Z, et al. A Big Bang model of human colorectal tumor growth. Nat Genet 2015;47:209-16.

44. Chen Z, Duldulao MP, Li W, et al. Molecular diagnosis of response to neoadjuvant chemoradiation therapy in patients with locally advanced rectal cancer. J Am Coll Surg 2011;212:1008-17.

45. Ondrejka SL, Schaeffer DF, Jakubowski MA, et al. Does neoadjuvant therapy alter KRAS and/or MSI results in rectal adenocarcinoma testing? Am J Surg Pathol 2011;35:132730.

46. Jo P, König A, Schirmer M, et al. Heterogeneity of KRAS mutation status in rectal cancer. PLoS One 2016;11:e0153278.

47. Boissiere-Michot F, Lopez-Crapez E, Frugier H, et al. KRAS genotyping in rectal adenocarcinoma specimens with low tumor cellularity after neoadjuvant treatment. Mod Pathol 2012;25:731-9.

48. Gollins S, West N, Sebag-Montefiore D, et al. Preoperative chemoradiation with capecitabine, irinotecan and cetuximab in rectal cancer: significance of pre-treatment and post-resection RAS mutations. Br J Cancer 2017;117:1286-94. 
49. Sclafani F, Chau I, Cunningham D, et al. KRAS and BRAF mutations in circulating tumour DNA from locally advanced rectal cancer. Sci Rep 2018;8:1445.

\section{Acknowledgements}

This work was supported by the NIHR BRC at The Royal Marsden NHS Foundation Trust and The Institute of Cancer Research. The EXPERT study was supported by a fellowship grant from the Pelican Cancer Foundation and by an education grant from Sanofi-Aventis which also provided the study drug. The EXPERT-C trial was endorsed by Cancer Research UK and was supported by a research grant from Merck \& Co. Sanofi-Aventis and Merck \& Co. provided the study drugs. Neither company was involved in study design, data analysis, or manuscript preparation or had access to study data.

\section{Figure legends}

Figure 1. Study flow diagram

Figure 2. Progression-free survival by TP53 and KRAS/NRAS status 

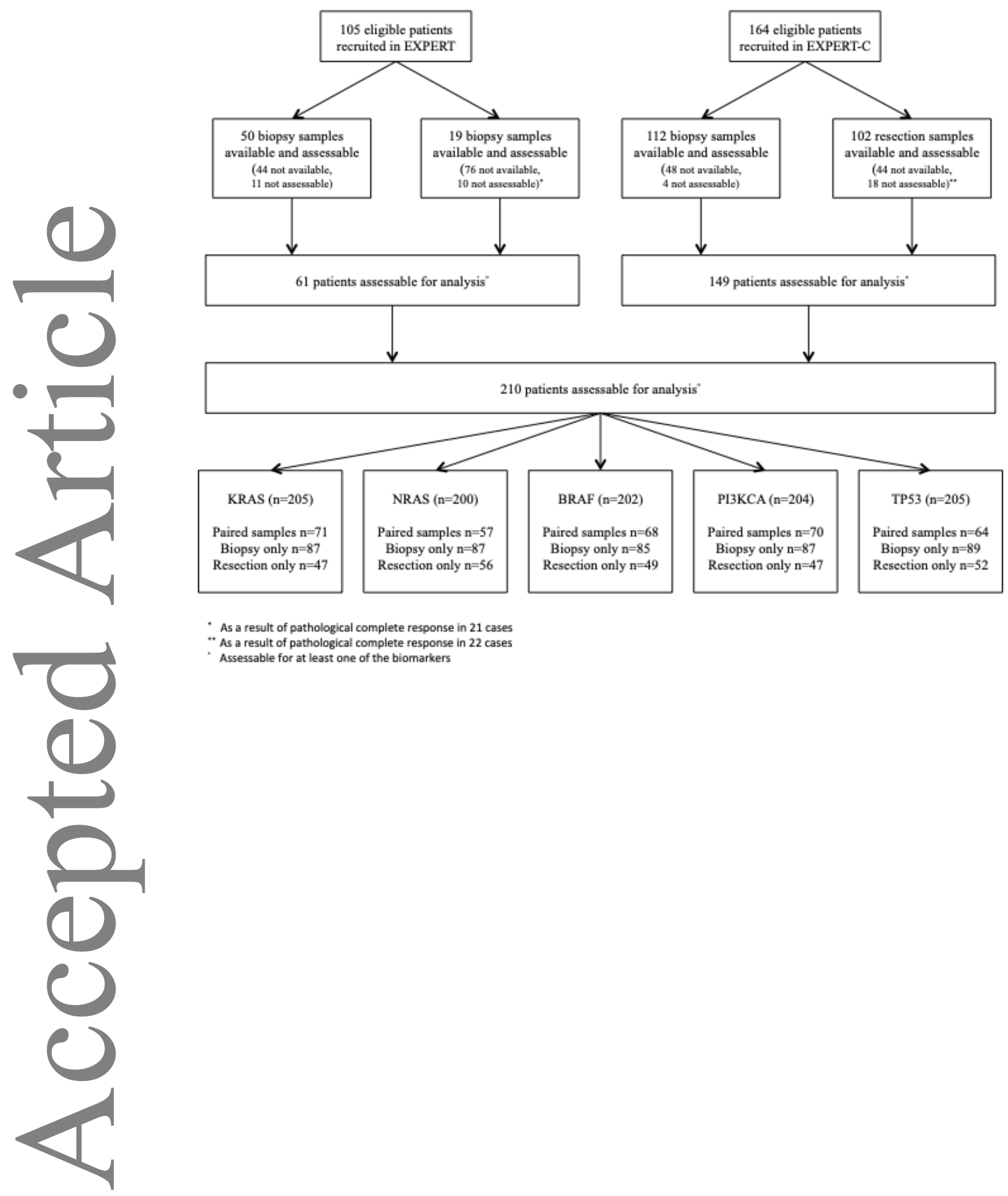

- As a result of pathological complete response in 21 cases

* As a result of pathological complete response in 22 cases

Assessable for at least one of the biomarkers 

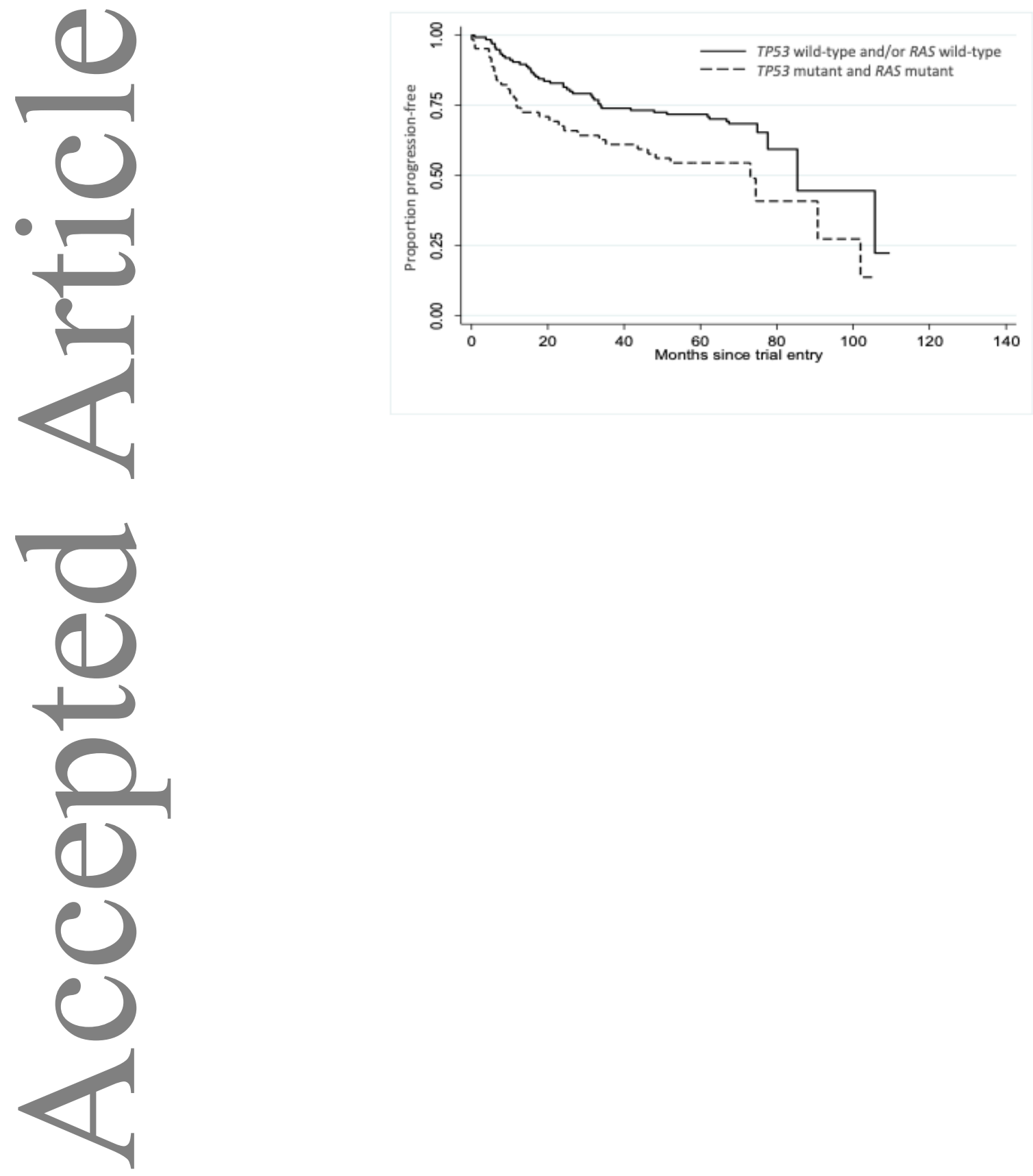

This article is protected by copyright. All rights reserved 
Table 1. Mutation rates in biopsy and resection samples, and overall number of patients with mutant tumours

\begin{tabular}{|c|c|c|c|}
\hline Gene & $\begin{array}{c}\text { Biopsy samples } \\
\text { harbouring mutations }\end{array}$ & $\begin{array}{c}\text { Resection samples } \\
\text { harbouring mutations }\end{array}$ & $\begin{array}{c}\text { Overall number of } \\
\text { patients with mutant } \\
\text { tumours }\end{array}$ \\
\hline$K R A S$ & $72 / 158(46 \%)$ & $35 / 118(30 \%)$ & $89 / 205(43 \%)$ \\
\hline NRAS & $13 / 144(9 \%)$ & $6 / 113(5 \%)$ & $17 / 200(9 \%)$ \\
\hline$B R A F$ & $7 / 153(5 \%)$ & $2 / 117(2 \%)$ & $7 / 202(4 \%)$ \\
\hline$P I 3 K C A$ & $17 / 157(11 \%)$ & $4 / 117(3 \%)$ & $18 / 204(9 \%)$ \\
\hline$T P 53$ & $98 / 153(64 \%)$ & $46 / 116(40 \%)$ & $123 / 205(60 \%)$ \\
\hline
\end{tabular}


Table 2. Mutation concordance between biopsy and resection in patients with paired specimens

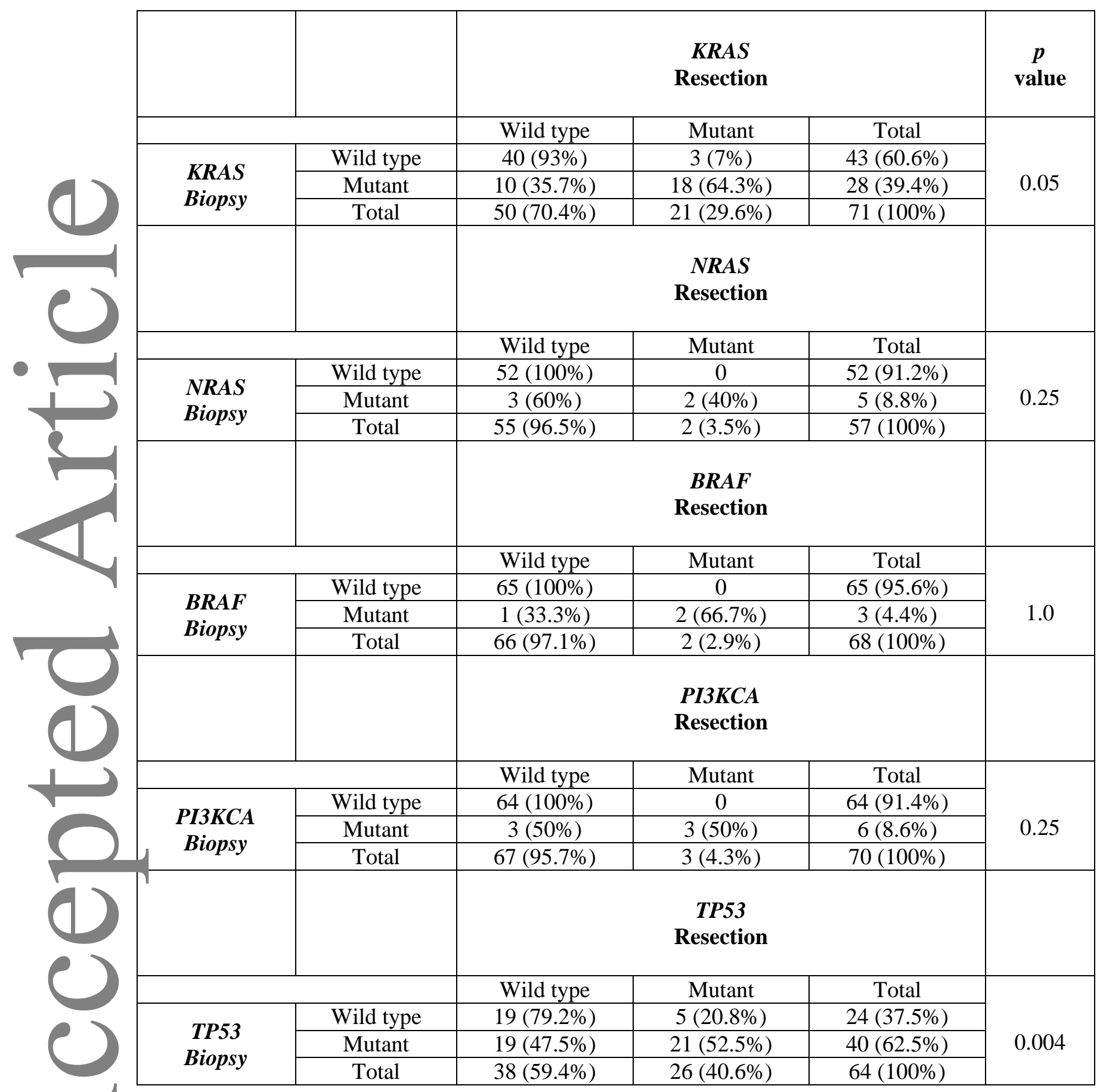

${ }^{*}$ Mc Nemar's test 
Table 3. Patient outcomes by single gene mutational status

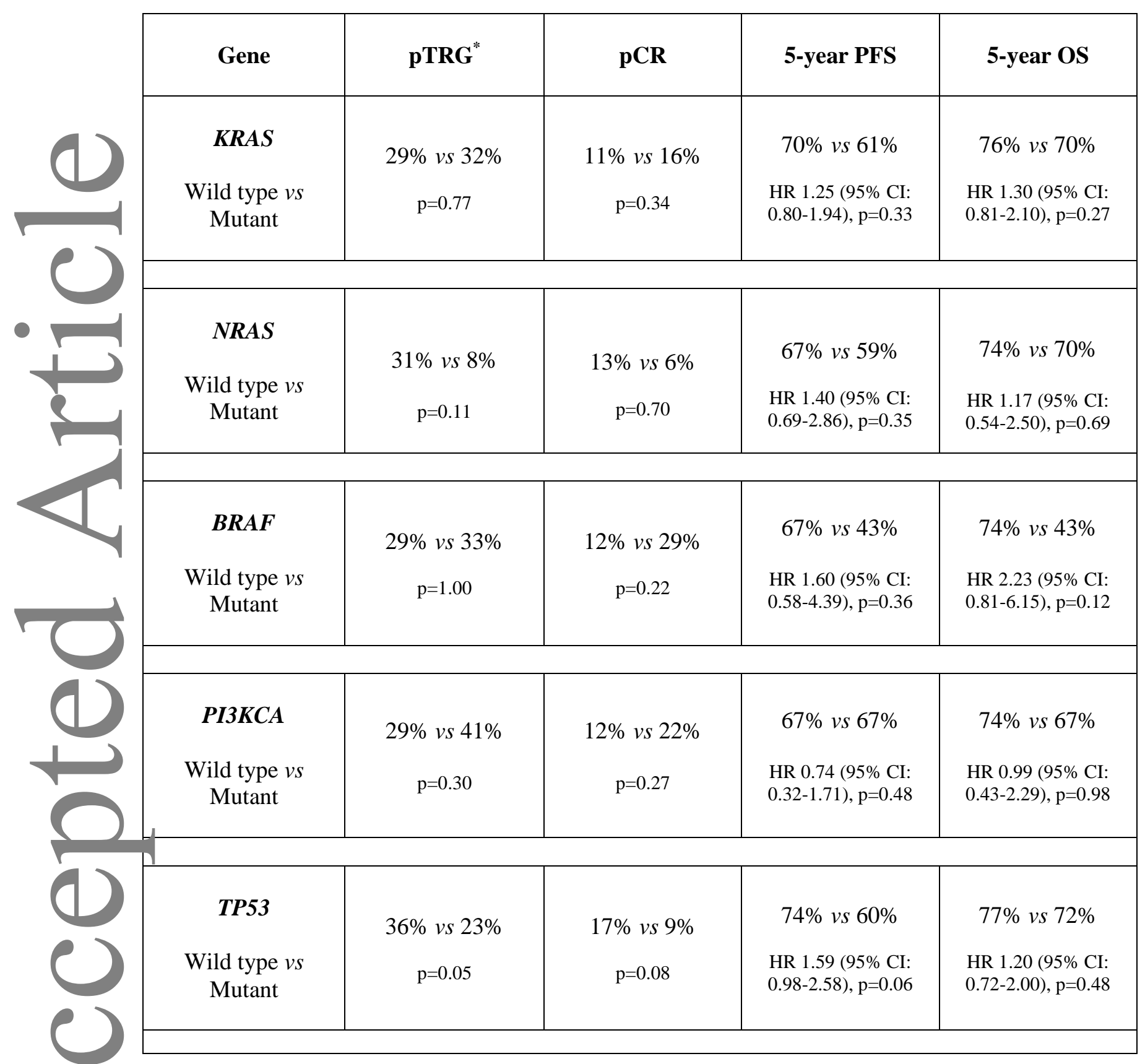

*Includes TRG 3 and 4 according to Dworak et al.

Abbreviations: CI: confidence intervals; HR: hazard ratio; pCR: pathological complete response; PFS: progression-free survival; pTRG: pathological tumour regression; OS: overall survival. 
3:45 PM DEC 3, 2019

THE MOMENT YOU GAIN

COMPLETE UNDERSTANDING

OF THE CELL

$+$
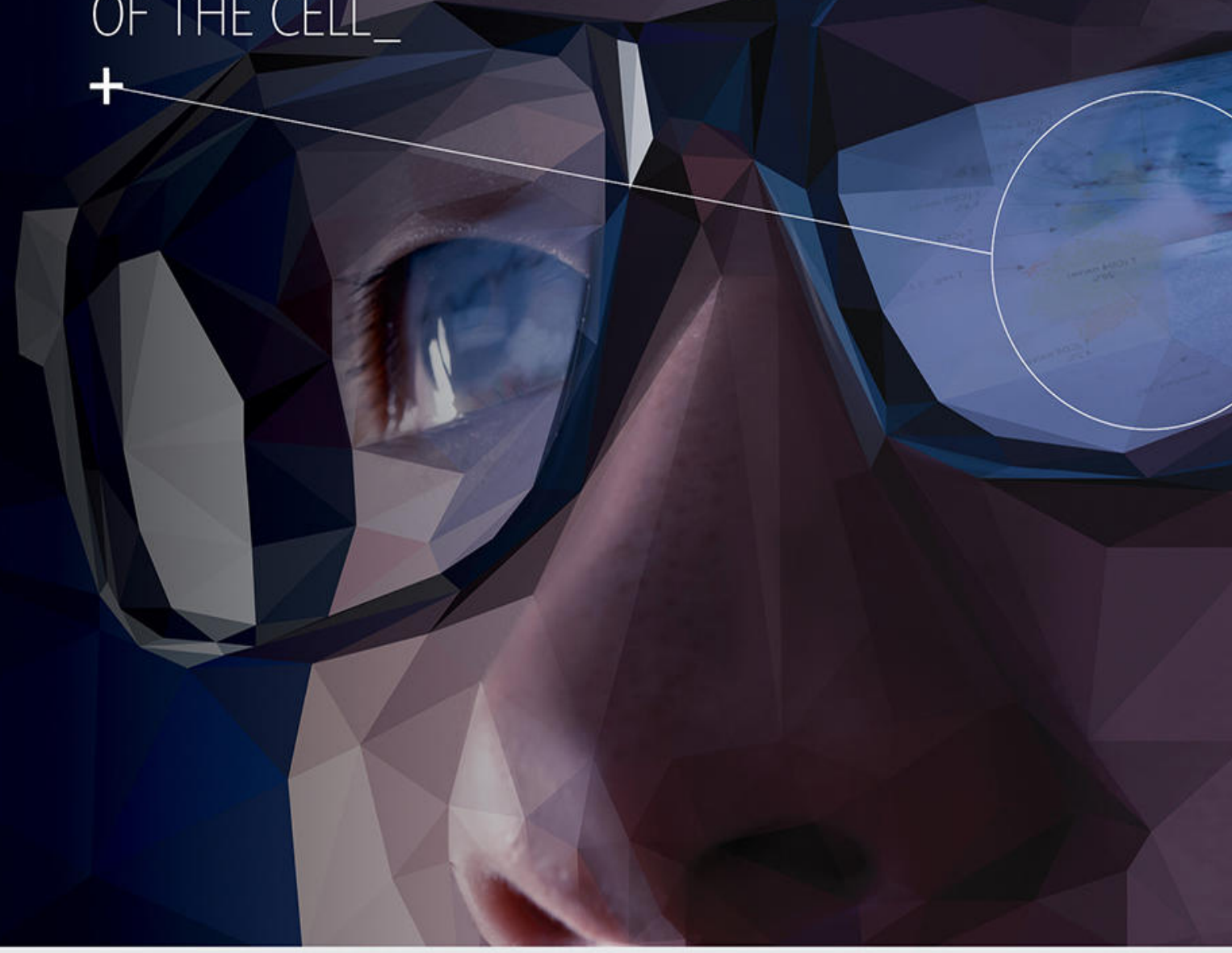

\section{THE DIFFERENCE OF}

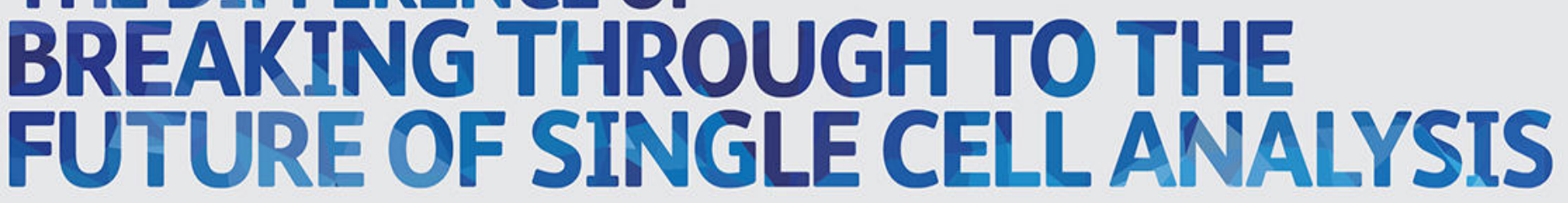

PROPELLING SCIENTISTS TO DEEPER UNDERSTANDING WITH A COMPLETE WORKFLOW SOLUTION.

The $\mathrm{BD}^{\odot} \mathrm{AbSeq}$ and $\mathrm{BD}$ Rhapsody ${ }^{\mathrm{Tm}}$ single-cell analysis system bring the future of immunology and oncology research to your fingertips. Together with the robust SeqGeq ${ }^{\text {TM }}$ analysis software, you can harness the power of single cell multiomics by simultaneously analyzing protein biomarkers and RNA. Offering customizable assays and incredible efficiency from profiling thousands of single cells in a workflow, our system reduces experimentation time and sequencing costs. Discover groundbreaking technology to help you push past the limits of single cell analysis. Discover the new BD. 